\section{Are Trojans pursuing the eclipsing pulsar?}

SIR-In this issue, Fruchter et al. ${ }^{1}$ report the discovery of a pulsar in an eclipsing binary system. The pulsar is moving in a nearly circular orbit (eccentricity $<0.001)$. The orbital parameters suggest that the companion has a very low mass, much lower than the probable masses of the companions of other known radio and $\mathrm{X}$-ray binary pulsars. Interestingly, the mass is sufficiently low that the Lagrange points $L_{4}$ and $L_{5}$ of the restricted threebody problem are points of stable equilibrium. There is thus the possibility that smaller agglomerations of matter are orbiting the system near these points. The situation could be analogous to that of the Trojan asteroids, which oscillate about $\mathrm{L}_{4}$ and $L_{5}$ in the Sun-Jupiter system.

The pulsar mass function is

$$
f=\frac{M_{1} q^{3} \sin ^{3} i}{(1+q)^{2}}=5.20 \times 10^{-6} M_{\odot}
$$

where $M_{1}$ is the pulsar mass, $i$ is the orbital inclination angle and $q=M_{2} / M_{1}$, where $M_{2}$ is the companion mass. The Lagrange points are stable provided $q \leq q_{\text {cri: }}=$ 0.0385 (see, for example, ref. 2). From equation (1), this will be true provided $\sin i \geq \sin i_{\text {стіi }}=0.412\left(M_{1} / 1.4 M_{\odot}\right)^{-1 / 3}$. For a pulsar of mass $1.4 M_{\odot}$, this constraint requires $i>i_{\text {crit }}=24^{\circ}$, which is almost certainly guaranteed by the presence of an eclipse. The dependence of this result on the assumed pulsar mass is very weak. Only a pulsar of mass $\leqslant 0.1 M_{\odot}$ could be eclipsed and yet have unstable Lagrange points. (Note that the minimum mass for a neutron star is $0.1 M_{\odot}$ (see, for example, ref. 3).) In fact, for the likely values $\sin i=$ $1, M_{1}=1.4 M_{\odot}$, the companion mass is $M_{2}$ $=0.022 M_{\odot}$ and the mass ratio $q$ is $1 / 64$, well within the stability range for the Lagrange points.

The eclipse is centred on orbital phase 0.25 , when the companion is between the pulsar and the Earth. The orbital phases of $\mathrm{L}_{4}$ and $\mathrm{L}_{5}$ are at $0.25 \pm \theta / 2 \pi$, where $\theta$ $=\tan ^{-1}[\sqrt{3}(1+q) /(1-q)]$.

Because the range of $q$ for stable Lagrange points is small, the corresponding range in phase is small. As $q$ varies from 0 to $q_{\text {crit }}$, the phase of one Lagrange point varies from 0.42 to 0.44 , while the phase of the other varies from 0.08 to 0.06 . The detection of matter at these phases would confirm that the mass ratio is below $q_{\text {crit }}$. Because of the weak dependence on $q$, however, it is unlikely that a precise mass ratio could be determined. Moreover, matter would probably librate about the

1. Fruchter, A.S., Stinebring, D.R. \& Taylor, J.H. Nature 333, 237-239 (1988)

2. Danby, J.M.A. Fundamentals of Celestial Mechanics (Macmillan, New York, 1962).

3. Shapiro, S.L. \& Teukolsky. S.A. Black Holes, White Dw'arfs, and Neutron Stars: The Physics of Compact Obiects, Ch.9(Wiley, New York, 1983).

Lagrange points. Were it possible to

SiR-Several findings suggest that lefthandedness may be associated with

The relative survival rate of right- and lefthanders. Each point represents the difference between the cumulative percentage survival of the two groups. Points above the line, higher survival rates for right-handers; points below the line, higher survival rates for left-handers. Stars, ages at which there were no surviving left-handers.

we have analysed all baseball players listed in The Baseball Encyclopedia ${ }^{2}$ for whom dates of birth and death, as well as throwing and batting hand, are reported. A subject was assigned to a handedness group only when both throwing and batting hand were the same with no indicated change in hand use.

Mean age at death for the 1,472 righthanders was 64.64 years (s.d. $=15.5$ ) and 236 left-handers was 63.97 years (s.d. = 15.4). This difference is difficult to interpret as the range is so large and the distribution is skewed. However, a nonparametric test of group differences (WaldWolfowitz runs test) indicated that the greater longevity for right-handers is significant $(Z=6.63, P<0.001)$. To clarify the pattern of results, we also examined data for the cumulative proportion of individuals surviving at each age. We found that the groups are virtually identical in mortality until the age of 33 . From track the orbit of such libration, or even to measure the period, the mass ratio could then be determined ${ }^{2}$.

This binary system is most probably still very active. There may be a powerful beam of high-energy particles from the pulsar and a strong stellar wind from the companion'. Thus it is very possible that nothing will be found at either of the Lagrange points, even if they are stable.

\section{Do right-handers live longer?} reduced longevity. For instance, Porac and Coren ' reported that 13 per cent of 20 year-olds are left-handed but only 5 per cent of those in their fifties and virtually nobody of 80 or above. We believe that this absence of left-handers from the oldest age groups reflects higher biological and environmental risk.

To investigate further the relationship between handedness and age of death,
On the other hand, this is the first system outside the Solar System where the conditions are favourable for observing matter at stable Lagrange points.

Stuart L. Shapiro Saul A. Teukolsky

\section{Center for Radiophysics and Space}

\section{Research and}

Departments of Astronomy and

Physics,

Cornell University, Ithaca,

New York 14853, USA

that age onwards about 2 per cent more right-handers than left-handers survive at each age $(P<0.001)$ (see figure).

Although these data suggest that lefthandedness is associated with reduced longevity, they do not provide any direct causal information. Three factors seem possible. First if prenatal and perinatal birth stressors are more probable in lefthanders ${ }^{1.36}$ it may reduce their ability to survive. Second, genetic effects and intrauterine hormones may have reduced the effectiveness of the immune system of sinistrals, increasing the likelihood of an earlier death ${ }^{7}$. Finally, left-handers may

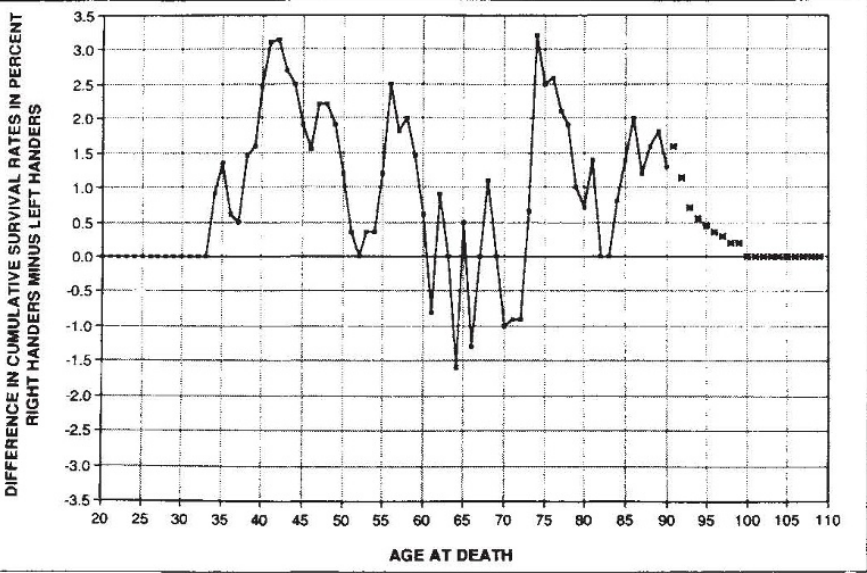

have more accidents in an environment designed for a right-handed majority'. These factors may act singly or in concert to account for the fact that left-handers seem to have a shorter life span.

Department of Psychology,

Diane F. Halpern

California State University,

San Bernardino,

California 92407, USA

Department of Psychology,

University of British Columbia,

Vancouver V6T 1 Y7, Canada

. Porac, C. \& Coren, S. Lateral Preferences and Human Behavior (Springer, New York, 1981).

. Reichler, J.L. (ed.) The Basebull Encyclopedia (Macmillan, New York, 1979).

Bakan. P. Nature. 229, $195(1971)$

Coren. S. \& Porac, C. Behav. Gienet. 10, 123-138 (1980)

S. Searleman, A. Porac, C. \& Coren, S. Can. J. Psychol. 36, $478(1982)$.

6. O'Callaghan, M.J. et al. Lancet i, 1155, (1987)

. Geschwind, N. \& Galaburda, A.M. Cerebral Lateralization: Biological Mechanisms, Associations, and Pathotogy (MIT. Cambridge, Massachusetts 1987). 